Environment Conservation Journal 15(1\&2) 35-47 , 2014

ISSN 0972-3099 (Print) 2278-5124 (Online)

Abstracted and Indexed

\title{
Phytodiversity (Angiosperms, Gymnosperms and Pteridophytes) in Lamberi forest range, Rajouri, J\&K, India
}

\author{
Jyoti Sharma, Anil K. Raina $\bowtie$ and Sachin Sharma
}

Received: 17.03.2014

Accepted: 13.05.2014

\begin{abstract}
The present study has been carried to assess phytodiversity in the Lamberi Forest Range of Rajouri, J\&K, India. A total of 222 species of angiosperms (217), gymnosperm (1) and pteridophytes (4) belonging to 80 families and 167 genera has been recorded. Among angiosperms, dicots have been represented by 203 species distributed in 152 genera and 69 families while the monocots have been represented by only 14 species distributed in 6 families and 10 genera. Overall, the ratio of family to genus has been worked out to be 1: 2.87 , family to species $1: 2.77$ while the genus to species has been worked out to be 1: 1.32. The dominant families of the area were Asteraceae, Lamiaceae, Fabaceae and Rosaceae. Forty families show monotypic representation.
\end{abstract}

Keywords: Angiosperm, Gymnosperm, Jammu, Lamberi forest range, Phytodiversity, Pteridophytes

\section{Introduction}

The knowledge of the floristic composition, species diversity and structural analysis studies of a plant community are prerequisite to understand the overall structure and function of any ecosystem and also are essential for providing information on species richness of the forests, forest management purpose and help in understanding forest ecosystem functions (Venu, 2002; Giriraj et al., 2008; Pappoe et al., 2010). Documenting basic patterns of biodiversity is fundamental for prioritizing areas for conservation and management action (Villasenor et al., 2007). As forests are known to be critically important habitats in terms of the biological diversity they contain and in terms of the ecological functions they serve by providing important environmental benefits (Bekele, 1994; SCBD, 2001; Vivero et al., 2005; Nune et al., 2010), knowledge of their structure and function is of utmost importance. The Indian Himalayan region occupies a special place in the mountain ecosystems of the world. This region is considered as the repository of biological and cultural diversity (Negi and Gaur, 1994). While the utilization of biological resources is a prerequisite for human

Author's Address

Deptt of Environmental Sciences, University of Jammu, Jammu, India

Email: anilkraina@yahoo.com sustenance on this planet, at the same time it is equally essential to conserve these resources for future generations through their sustainable utilization. Therefore, documentation of diversity of any region is of paramount importance as this will also help in better conservation, management and utilization of its resources. Study on forest composition, diversity and socio-economic status in Jammu region adjoining to the study area has been done by Singh, 2002; Sharma, 2003 and Raina and Kumar, 2011. Enumeration of Lamberi forest range which is very rich in plant diversity has not been done so far. Therefore, in this study we have tried to work out the floristic composition of this forest covering all the important aspects of the phytodiversity (angiosperms, gymnosperms and pteridophytes) of the Lamberi forest range.

\section{Study area}

Lamberi forest range (Latitude $33^{\circ} 06^{\prime}$ to $33^{0} 13^{\prime}$ North and Longitude $74^{0} 08^{\prime}$ to $74^{0} 18^{\prime}$ East) is a part of Nowshera forest division in Rajouri district of J\&K. This forest range covers an altitudinal range of $600 \mathrm{~m}$ to $1200 \mathrm{~m}$ a.s.l. and experiences four distinct seasons as Summer (Mid March to end of June), Monsoon (July to September), Autumn (October to mid November) and Winter (mid November to mid March). There is a great extreme 
of temperature with June recorded as hottest and January coldest month with average maximum and minimum temperature of $37.4^{0} \mathrm{C}$ to $7.42^{\circ} \mathrm{C}$. Average rainfall of area is $500 \mathrm{~mm}$. Most of the rainfall occurs during monsoon season.

\section{Material and Methods}

Extensive field surveys were conducted in the study area from June 2011 to May 2012, in different seasons to assess the diversity of higher plants including pteridophytes. Species were identified with the help of various local, regional and National floras and herbaria. Majority of the plants were identified on the spot by their vernacular names. Taxonomical categories-genera and species within the family were placed alphabetically.

\section{Results and Discussion}

The list of plants collected from the study area has been depicted in the Table 1 . The perusal of table revealed the presence of a total of 222 species belonging to 80 families and 167 genera. Dicots have been represented by 203 species distributed in 152 genera and 69 families which formed a major part of the flora while the monocots have been represented by only 14 species distributed in 6 families and 10 genera (Table 2). The pteridophytes have been represented by 4 genera belonging to 4 families viz. Adiantaceae, Dryopteradaceae, Pteridaceae and Thelypteridiaceae. In gymnosperms Pinaceae (1 species and 1 genera) was the only family which has been recorded from the study area.

Table 1: List of Plants Collected from Lamberi Forest Range along with their Life forms, Habit, Division and Familial description

\begin{tabular}{|c|c|c|c|}
\hline S. No & Name & Life form & Habit \\
\hline \multicolumn{4}{|c|}{ Angiosperm Dicot } \\
\hline \multicolumn{4}{|c|}{ Family : Acanthaceae } \\
\hline 1. & Barlera dichotoma Roxb. & $\mathrm{CH}$ & Herb \\
\hline 2. & Justicia adhatoda Nees. & $\mathrm{N}$ & Shrub \\
\hline 3. & Justica simplex D. Don & $\mathrm{CH}$ & Herb \\
\hline 4. & Strobilanthes alatus Nees. & $\mathrm{TH}$ & Herb \\
\hline \multicolumn{4}{|c|}{ Family :Amaranthaceae } \\
\hline 5. & Achyranthes aspera L. & $\mathrm{H}$ & Herb \\
\hline 6. & Aerva scandens wall. & $\mathrm{CH}$ & Herb \\
\hline 7. & Alysicarpus vaginalis (L.) DC. & TH & Herb \\
\hline 8. & Amaranthus gangeticus Linn. & $\mathrm{TH}$ & Herb \\
\hline 9. & Amaranthus viridis $\mathrm{L}$ & $\mathrm{TH}$ & Herb \\
\hline \multicolumn{4}{|c|}{ Family: Anacardiaceae } \\
\hline 10. & Lannea coromandelica Merr. & $\mathrm{M}$ & Tree \\
\hline 11. & Mangifera indica Linn. & $\mathrm{M}$ & Tree \\
\hline 12. & Rhus cotinus L. & $\mathrm{N}$ & Shrub \\
\hline \multicolumn{4}{|c|}{ Family: Apiaceae } \\
\hline 13. & Centella asiatica Urb. & $\mathrm{H}$ & Herb \\
\hline 14. & Scandix pectin-veneris Linn. & $\mathrm{TH}$ & Herb \\
\hline \multicolumn{4}{|c|}{ Family: Apocynaceae } \\
\hline 15. & Carissa opaca Stapf. & $\mathrm{N}$ & Shrub \\
\hline 16. & Nerium indicum Mill. & $\mathrm{N}$ & Shrub \\
\hline
\end{tabular}




\begin{tabular}{|c|c|c|c|}
\hline \multicolumn{4}{|c|}{ Family: Araliaceae } \\
\hline 17. & Hedera nepalensis K. Koch. & $\mathrm{L}$ & Climber \\
\hline \multicolumn{4}{|c|}{ Family: Arecaceae } \\
\hline 18. & Phoenix sylvestris (Linn.) Roxb. & $\bar{M}$ & Tree \\
\hline \multicolumn{4}{|c|}{ Family: Asclepiadaceae } \\
\hline 19. & Calotropis procera R.Br. & $\bar{N}$ & Shrub \\
\hline 20. & Perugularia extensa N.E.Br. & $\mathrm{L}$ & Climber \\
\hline \multicolumn{4}{|c|}{ Family: Asteraceae } \\
\hline 21. & $\begin{array}{l}\text { Anaphallis triplenervis Sims ex } \\
\text { C.B.Clarke. }\end{array}$ & $\mathrm{CH}$ & Herb \\
\hline 22. & Artimisia parviflora Roxb. ex D. Don. & $\mathrm{CH}$ & Herb \\
\hline 23. & Bidens biternata Linn. & $\mathrm{TH}$ & Herb \\
\hline 24. & Cirsium arvense (Linn) Scop. & $\mathrm{CH}$ & Herb \\
\hline 25. & $\begin{array}{l}\text { Cirsium wallichii DC. } \\
\text {. }\end{array}$ & $\mathrm{CH}$ & Herb \\
\hline 26. & Cichorium intybus L. & $\mathrm{H}$ & Herb \\
\hline 27. & Conzya japonicus (Thunb.) DC. & $\mathrm{TH}$ & Herb \\
\hline 28. & Cousinia falconeri Hook.F. & $\mathrm{TH}$ & Herb \\
\hline 29. & Erigeron canadensis $L$. & $\mathrm{TH}$ & Herb \\
\hline 30. & Gnaphalium indicum L. & $\mathrm{TH}$ & Herb \\
\hline 31. & Parthenium hysterophorus L. & $\mathrm{TH}$ & Shrub \\
\hline 32. & Saussurea albescens Hook.,f. & $\mathrm{H}$ & Herb \\
\hline 33. & $\begin{array}{l}\text { Saussurea heteromalla (D.Don) Hand.- } \\
\text { Mazz. }\end{array}$ & $\mathrm{H}$ & Herb \\
\hline 34. & Sonchus arvensis L. & $\mathrm{TH}$ & Herb \\
\hline 35. & Sonchus asper (L.)Hill & $\mathrm{TH}$ & Herb \\
\hline 36. & Taxaacum officinale Weber. & $\mathrm{TH}$ & Herb \\
\hline 37. & Trifolium arvense Linn. & $\mathrm{H}$ & Herb \\
\hline 38. & Trifolium pretense Linn. & $\mathrm{H}$ & Herb \\
\hline 39. & Trifolium repens Linn. & $\mathrm{H}$ & Herb \\
\hline 40. & Xanthium stromarium Linn. & $\mathrm{TH}$ & Herb \\
\hline \multicolumn{4}{|c|}{ Family: Balsaminaceae } \\
\hline 41. & Impatiens brachycentra Kar.\&Kir. & $\mathrm{TH}$ & Herb \\
\hline \multicolumn{4}{|c|}{ Family: Berberidaceae } \\
\hline 42. & Berberis lyceum Royle. & $\mathrm{N}$ & Shrub \\
\hline \multicolumn{4}{|c|}{ Family: Bignoniaceae } \\
\hline 43. & Bougainvillea glabra Choisy. & $\mathrm{L}$ & Climber \\
\hline \multicolumn{4}{|c|}{ Family: Bombaceae } \\
\hline 44. & Bombax ceiba L. & $\mathrm{M}$ & Tree \\
\hline
\end{tabular}




\begin{tabular}{|c|c|c|c|}
\hline \multicolumn{4}{|c|}{ Family: Boraginaceae } \\
\hline 45. & Cordia mуха L. & $\mathrm{M}$ & Tree \\
\hline 46. & Heliotropium elipticum Ledeb. & $\mathrm{TH}$ & Herb \\
\hline \multicolumn{4}{|c|}{ Family: Brassicaceae } \\
\hline 47. & Lepidium sativum Linn. & $\mathrm{TH}$ & Herb \\
\hline \multicolumn{4}{|c|}{ Family: Buddlejaceae } \\
\hline 48. & Buddleja asiatica Lour. & $\bar{N}$ & Shrub \\
\hline \multicolumn{4}{|c|}{ Family: Cactaceae } \\
\hline 49. & Opuntia vulgaris Mill. & $\mathrm{N}$ & Shrub \\
\hline \multicolumn{4}{|c|}{ Family: Caesalpinaceae } \\
\hline 50. & Cassia fistula $\mathrm{L}$. & $\mathrm{M}$ & Tree \\
\hline 51. & Cassia occidentalis L. & $\mathrm{TH}$ & Herb \\
\hline 52. & Cassia tora L. & $\mathrm{TH}$ & Herb \\
\hline 53. & Bauhinia purpurea Linn. & $\mathrm{M}$ & Tree \\
\hline 54. & Bauhinia variegata Linn. & $\bar{M}$ & Tree \\
\hline \multicolumn{4}{|c|}{ Family: Cannabinaceae } \\
\hline 55. & Cannabis sativa Linn. & $\mathrm{CH}$ & Herb \\
\hline \multicolumn{4}{|c|}{ Family: Caryophyllaceae } \\
\hline 56. & Silene conoida Linn. & $\mathrm{TH}$ & Herb \\
\hline 57. & Silene inflata Sm. & $\mathrm{TH}$ & Herb \\
\hline 58. & Stellaria media (L.)Vill. & $\mathrm{TH}$ & Herb \\
\hline 59. & Stellaria asiatica Mill. & $\mathrm{TH}$ & Herb \\
\hline \multicolumn{4}{|c|}{ Family: Celastraceae } \\
\hline 60. & Gymnosporia royleana Laws. & $\mathrm{N}$ & Shrub \\
\hline \multicolumn{4}{|c|}{ Family: Chenopodiaceae } \\
\hline 61. & Chenopodium murale L. & $\mathrm{TH}$ & Herb \\
\hline \multicolumn{4}{|c|}{ Family: Clusiaceae } \\
\hline 62. & Hypericum cernuum Roxb. & $\mathrm{CH}$ & Herb \\
\hline \multicolumn{4}{|c|}{ Family: Combretaceae } \\
\hline 63. & Terminalia chebula Retz. & $\mathrm{M}$ & Tree \\
\hline \multicolumn{4}{|c|}{ Family: Commelianaceae } \\
\hline 64. & Commelina bengalensis L. & $\mathrm{TH}$ & Herb \\
\hline 65. & Commelina communis Linn. & $\mathrm{TH}$ & Herb \\
\hline \multicolumn{4}{|c|}{ Family: convolvulaceae } \\
\hline 66. & Ipomoea carnea Jacq. & $\mathrm{CH}$ & Shrub \\
\hline 67. & Ipomoea eriocarpa R.Br. & $\mathrm{L}$ & climber \\
\hline 68. & Ipomoea muricata Jacq. & $\mathrm{L}$ & Climber \\
\hline 69. & Ipomoea nil Roth. & $\mathrm{L}$ & Climber \\
\hline
\end{tabular}




\begin{tabular}{|c|c|c|c|}
\hline 70. & Ipomoea purpurea (Linn) Roth. & $\mathrm{L}$ & Climber \\
\hline \multicolumn{4}{|c|}{ Family: Cucurbitaceae } \\
\hline 71. & Tridax procumbens L. & $\mathrm{TH}$ & Herb \\
\hline \multicolumn{4}{|c|}{ Family: Cuscutaceae } \\
\hline 72. & Cuscuta reflexa Roxb. & $\mathrm{E}$ & Epiphyte \\
\hline \multicolumn{4}{|c|}{ Family: Cyperaceae } \\
\hline 73. & Carex spp. & $\mathrm{H}$ & Herb \\
\hline 74. & Eriophorum comosum Nees. & $\mathrm{CH}$ & Herb \\
\hline \multicolumn{4}{|c|}{ Family: Ebenaceae } \\
\hline 75. & Diospyros cordifolia Roxb. & $\mathrm{M}$ & Tree \\
\hline \multicolumn{4}{|c|}{ Family: Euphorbiacaea } \\
\hline 76. & Phyllanthus emblica L. & $\mathrm{M}$ & Tree \\
\hline 77. & Euphorbia geniculata Orteg. & $\mathrm{TH}$ & Herb \\
\hline 78. & Euphorbia helioscopa L. & $\mathrm{TH}$ & Herb \\
\hline 79. & Euphorbia hirta L. & $\mathrm{TH}$ & Herb \\
\hline 80. & Euphorbia prunifolia Jacq. & $\mathrm{TH}$ & Herb \\
\hline 81. & Euphorbia royleana Boiss. & $\mathrm{N}$ & Shrub \\
\hline 82. & Mallotus philippensis Muell. Arg. & $\mathrm{M}$ & Tree \\
\hline 83. & Ricinus communis L. & $\mathrm{N}$ & Shrub \\
\hline \multicolumn{4}{|c|}{ Family: Fabaceae } \\
\hline 84. & Dalbergia sissoo Roxb. & $\bar{M}$ & Tree \\
\hline 85. & Desmodium trifolium Dc. & $\mathrm{CH}$ & Herb \\
\hline 86. & Erythrina indica Lam. & $\mathrm{M}$ & Tree \\
\hline 87. & Indigofera pulchella Roxb. & $\mathrm{N}$ & Shrub \\
\hline 88. & Indigofera linifolia (L.f.) Retz & $\mathrm{TH}$ & Herb \\
\hline 89. & Lathyrus sativus Linn & $\mathrm{H}$ & Herb \\
\hline 90. & Meliotus alba Medic. & $\mathrm{TH}$ & Herb \\
\hline 91. & Parkinsonia aculeate L. & $\mathrm{M}$ & Tree \\
\hline 92. & Vicia sativa Linn. & $\mathrm{C}$ & Climber \\
\hline \multicolumn{4}{|c|}{ Family: Flacourtiaceae } \\
\hline 93. & Flacourtia indica Merr & $\mathrm{M}$ & Tree \\
\hline \multicolumn{4}{|c|}{ Family: Fumariaceae } \\
\hline 94. & Fumaria indica Pugsley. & $\mathrm{TH}$ & Herb \\
\hline \multicolumn{4}{|c|}{ Family: Gentianaceae } \\
\hline 95. & Gentiana argentea $L$. & $\mathrm{H}$ & Herb \\
\hline \multicolumn{4}{|c|}{ Family: Geraniaceae } \\
\hline 96. & Geranium ocellatum Jacq. & $\mathrm{CH}$ & Herb \\
\hline 97. & Geranium rotundifolium L. & $\mathrm{CH}$ & Herb \\
\hline
\end{tabular}




\begin{tabular}{|c|c|c|c|}
\hline \multicolumn{4}{|c|}{ Family: Lamiaceae } \\
\hline 98. & Ajuga bracteosa Benth. & $\mathrm{TH}$ & Herb \\
\hline 99. & Ajuga parviflora Benth. & $\mathrm{H}$ & Herb \\
\hline 100. & Colebrookia oppositifolia Smith & $\mathrm{N}$ & Shrub \\
\hline 101. & Lamium album Linn. & $\mathrm{TH}$ & Herb \\
\hline 102. & Mentha longifolia Huds. & $\mathrm{HH}$ & Herb \\
\hline 103. & Mentha sylvestris L. & $\mathrm{HH}$ & Herb \\
\hline 104. & Mentha piperata L. & $\overline{\mathrm{H}}$ & Herb \\
\hline 105. & Nepeta hindostana (Roth)Haines & $\mathrm{CH}$ & Herb \\
\hline 106. & Nepeta spicata Wall. & $\mathrm{CH}$ & Herb \\
\hline 107. & Ocimum americana L. & $\mathrm{TH}$ & Herb \\
\hline 108. & Salvia lanata Roxb. & $\mathrm{CH}$ & Herb \\
\hline 109. & Stachys sericea Wall. & $\mathrm{TH}$ & Herb \\
\hline 110. & Vitex negundo Linn. & $\mathrm{N}$ & Shrub \\
\hline 111. & Vitex trifolia Linn. & $\mathrm{N}$ & Shrub \\
\hline \multicolumn{4}{|c|}{ Family: Lauracaea } \\
\hline 112. & Litsea umbrosa Nees. & $\bar{M}$ & Tree \\
\hline \multicolumn{4}{|c|}{ Family: Liliaceae } \\
\hline 113. & Gloriosa superba L. & $\mathrm{L}$ & Climber \\
\hline 114. & Reinwardtia indica Dumort. & $\mathrm{CH}$ & Herb \\
\hline \multicolumn{4}{|c|}{ Family: Linderniaceae } \\
\hline 115. & Lindernia ciliata (Colsmann) Pennell & $\mathrm{TH}$ & Herb \\
\hline \multicolumn{4}{|c|}{ Family: Lythraceae } \\
\hline 116. & Lagerstroemia parviflora (L.) Pers & $\mathrm{M}$ & Tree \\
\hline 117. & Woodfordia fruticosa kurz. & $\mathrm{N}$ & Shrub \\
\hline \multicolumn{4}{|c|}{ Family: Malvaceae } \\
\hline 118. & Malvestrum coromandelianum Garcke & $\mathrm{TH}$ & Herb \\
\hline 119. & Sida cordata Borssum. & $\mathrm{TH}$ & Herb \\
\hline \multicolumn{4}{|c|}{ Family: Meliaceae } \\
\hline 120. & Azadirachta indica A.Juss. & $\mathrm{M}$ & Tree \\
\hline 121. & Melia azedarach L. & $\mathrm{M}$ & Tree \\
\hline 122. & Toona ciliata M.Roemer. & $\bar{M}$ & Tree \\
\hline \multicolumn{4}{|c|}{ Family: Mimosaceae } \\
\hline 123. & Acacia catechu Wild. & $\mathbf{M}$ & Tree \\
\hline 124. & Acacia modesta Wild. & M & Tree \\
\hline
\end{tabular}


Phytodiversity (Angiosperms, Gymnosperms and Pteridophytes)

\begin{tabular}{|c|c|c|c|}
\hline 125. & Acacia nilotica (L.) Del. & $\mathrm{M}$ & Tree \\
\hline 126. & Albizia lebbeck (L.) Benth. & $\bar{M}$ & Tree \\
\hline 127. & Mimosa rubicaulis Lamk. & $\mathrm{N}$ & Shrub \\
\hline \multicolumn{4}{|c|}{ Family: Moraceae } \\
\hline 128. & Ficus auriculata Lour. & $\mathrm{M}$ & Tree \\
\hline 129. & Ficus benghalensis L.s & $\mathrm{M}$ & Tree \\
\hline 130. & Ficus carica L. & $\mathrm{M}$ & Tree \\
\hline 131. & Ficus hispida L.f. & $\bar{M}$ & Tree \\
\hline 132. & Ficus palmata Forssk. & $\mathrm{M}$ & Tree \\
\hline 133. & Ficus racemosa L. & $\bar{M}$ & Tree \\
\hline 134. & Ficus religiosa $L$ & $\mathrm{M}$ & Tree \\
\hline 135. & Morus alba L. & $\mathrm{M}$ & Tree \\
\hline 136. & Morus serrata Roxb. & $\bar{M}$ & Tree \\
\hline \multicolumn{4}{|c|}{ Family: Musaceae } \\
\hline 137. & Musa paradisiaca Linn. & $\mathrm{M}$ & Tree \\
\hline \multicolumn{4}{|c|}{ Family: Myrtaceae } \\
\hline 138. & Callistemon citrinus Skeels. & $\mathrm{M}$ & Tree \\
\hline 139. & Euclaptus tereticornis Sm. & $\bar{M}$ & Tree \\
\hline 140. & Psidium guajava L. & $\mathrm{M}$ & Tree \\
\hline 141. & Syzygium cumini (L.) Skeels & $\mathrm{M}$ & Tree \\
\hline \multicolumn{4}{|c|}{ Family: Nyctaginaceae } \\
\hline 142. & Boehmeria platyphylla D.Don. & $\mathrm{N}$ & Shrub \\
\hline 143. & Mirabilis jalapa L. & $\mathrm{CH}$ & Herb \\
\hline \multicolumn{4}{|c|}{ Family: Oleaceae } \\
\hline 144. & Jasminum officinale L. & $\mathrm{N}$ & Shrub \\
\hline 145. & Olea cuspidata Wall. ex G. Don & $\mathrm{M}$ & Tree \\
\hline \multicolumn{4}{|c|}{ Family: Onagraceae } \\
\hline 146. & Oenethera rosea Ait. & $\mathrm{TH}$ & Herb \\
\hline \multicolumn{4}{|c|}{ Family: Oxalidaceae } \\
\hline 147. & Oxalis corniculata $\mathrm{L}$. & $\overline{\mathrm{CH}}$ & Herb \\
\hline \multicolumn{4}{|c|}{ Family: Papaveraceae } \\
\hline 148. & Argimone mexicana L. & $\mathrm{TH}$ & Herb \\
\hline \multicolumn{4}{|c|}{ Family: Polygonaceae } \\
\hline 149. & Polygonum glabrum Willd. & $\mathrm{TH}$ & Herb \\
\hline 150. & Rumex dentatus Linn. & $\mathrm{TH}$ & Herb \\
\hline 151. & Rumex hastatus D. Don. & $\overline{\mathrm{CH}}$ & Herb \\
\hline
\end{tabular}




\begin{tabular}{|c|c|c|c|}
\hline 152. & Anagallis arvensis L. & $\mathrm{TH}$ & Herb \\
\hline 153. & Androsa rotundifolia Hardw. & $\mathrm{H}$ & Herb \\
\hline \multicolumn{4}{|c|}{ Family: Proteaceae } \\
\hline 154. & Grevillea robusta A. Cunn. Ex R.Br. & $\bar{M}$ & Tree \\
\hline \multicolumn{4}{|c|}{ Family: Punicaceae } \\
\hline 155. & Punica granatum Linn. & $\mathrm{N}$ & Shrub \\
\hline \multicolumn{4}{|c|}{ Family: Ranunculaceae } \\
\hline 156. & Anemone falconeri Thoms. & $\overline{\mathrm{H}}$ & Herb \\
\hline 157. & Delphinium uncinatum Hook.f. & $\bar{G}$ & Herb \\
\hline 158. & Rananculus aquatilis L. & $\mathrm{HH}$ & Herb \\
\hline 159. & Rananculus arvensis Linn. & $\mathrm{TH}$ & Herb \\
\hline 160. & Rananculus muricatus L. & $\mathrm{TH}$ & Herb \\
\hline 161. & Thallictrum pendunculatus Edgew. & $\overline{\mathrm{CH}}$ & Herb \\
\hline \multicolumn{4}{|c|}{ Family: Rhamnaceae } \\
\hline 162. & Rhamnus triquetra Wall. & $\mathrm{M}$ & Tree \\
\hline 163. & Sageretia oppositifolia Wall.ex Roxb. & $\mathrm{N}$ & Shrub \\
\hline 164. & $\begin{array}{l}\text { Zizyphus nummularia (Burm.f.) } \\
\text { Wight\&Arn }\end{array}$ & $\mathrm{N}$ & Shrub \\
\hline 165. & Zizyphus mauritiana Lamk. & $\mathrm{M}$ & Tree \\
\hline 166. & Zizyphus oxyphylla Edgew. & $\mathrm{M}$ & Tree \\
\hline \multicolumn{4}{|c|}{ Family: Rosaceae } \\
\hline 167. & Duchesnea indica (Andrews) Focke. & $\mathrm{CH}$ & Herb \\
\hline 168. & Potentilla supine L. & $\mathrm{H}$ & Herb \\
\hline 169. & Prinsepa utilis Royle. & $\mathrm{N}$ & Shrub \\
\hline 170. & Prunus domestica Linn. & $\mathrm{M}$ & Tree \\
\hline 171. & Prunus persica Linn. & $\mathrm{M}$ & Tree \\
\hline 172. & Pyrus communis Linn. & $\mathrm{M}$ & Tree \\
\hline 173. & Pyrus pashia Buch-Ham. & $\mathrm{M}$ & Tree \\
\hline 174. & Rosa multiflora Lindl. & $\mathrm{N}$ & Shrub \\
\hline 175. & Rubus ellipticus Smith. & $\mathrm{N}$ & Shrub \\
\hline \multicolumn{4}{|c|}{ Family: Rubiaceae } \\
\hline 176. & Gallium rotundifolium $L$. & $\mathrm{TH}$ & Herb \\
\hline 177. & Randia dumetorum Lamk. & $\mathrm{N}$ & Shrub \\
\hline 178. & Randia tetrasperma Benth. \& Hook & $\mathrm{N}$ & Shrub \\
\hline 179. & Rubia cordifolia L. & $\overline{\mathrm{H}}$ & Herb \\
\hline
\end{tabular}




\begin{tabular}{|c|c|c|c|}
\hline \multicolumn{4}{|c|}{ Family: Rutaceae } \\
\hline 180. & Aegle marmelos Corr. & $\mathrm{M}$ & Tree \\
\hline 181. & Zanthoxylam armatum DC. & $\mathrm{M}$ & Tree \\
\hline \multicolumn{4}{|c|}{ Family: Salicaceae } \\
\hline 182. & Populus ciliataWall ex Royle. & $\mathrm{M}$ & Tree \\
\hline 183. & Salix tetrasperma Roxb. & $\mathrm{M}$ & Tree \\
\hline \multicolumn{4}{|c|}{ Family: Sapindaceae } \\
\hline 184. & Dodonaea viscosaJacq. & $\mathrm{N}$ & Shrub \\
\hline 185. & Sapindus mukorosii Gaertn. & $\mathrm{M}$ & Tree \\
\hline \multicolumn{4}{|c|}{ Family: Scrophulariaceae } \\
\hline 186. & Mazus japonicus Kuntz. & $\mathrm{TH}$ & Herb \\
\hline 187. & Striga asiatica (L.) Kuntze. & $\mathrm{TH}$ & Herb \\
\hline 188. & Verbascum thapsus Linn. & $\mathrm{CH}$ & Herb \\
\hline 189. & Veronica agretis Linn. & $\mathrm{TH}$ & Herb \\
\hline \multicolumn{4}{|c|}{ Family: Solanaceae } \\
\hline 190. & Datura innoxia Mill. & $\mathrm{TH}$ & Herb \\
\hline 191. & Datura stromanium $L$. & $\mathrm{TH}$ & Herb \\
\hline 192. & Solanum erianthum D. Don & $\mathrm{N}$ & Shrub \\
\hline 193. & Solanum nigrum Linn. & $\mathrm{TH}$ & Herb \\
\hline 194. & Solanum pseudo-capsicum Linn. & $\mathrm{CH}$ & Herb \\
\hline 195. & \begin{tabular}{|l} 
Solanum surattense Burm.f \\
\end{tabular} & $\mathrm{TH}$ & Herb \\
\hline 196. & Solanum viarum Dunal. & $\overline{\mathrm{CH}}$ & Herb \\
\hline \multicolumn{4}{|c|}{ Family: Tiliaceae } \\
\hline 197. & Grewia optiva J.R Drumm. & $\mathrm{M}$ & Tree \\
\hline 198. & $\begin{array}{l}\text { Grewia oppositifolia Buch.-Ham. } \\
\text { ex Roxb. }\end{array}$ & $\mathrm{M}$ & Tree \\
\hline \multicolumn{4}{|c|}{ Family: Ulmaceae } \\
\hline 199. & Celtis australis Linn. & $\mathrm{M}$ & Tree \\
\hline \multicolumn{4}{|c|}{ Family: Utricaceae } \\
\hline 200. & Debregeasia spp. & $\mathrm{N}$ & Shrub \\
\hline \multicolumn{4}{|c|}{ Family: Verbenaceae } \\
\hline 201. & Lantana camara L. & $\mathrm{N}$ & Shrub \\
\hline \multicolumn{4}{|c|}{ Family: Violaceae } \\
\hline 202. & Viola odorata Linn. & $\mathrm{H}$ & Herb \\
\hline 203. & Viola patrinii L. & $\mathrm{H}$ & Herb \\
\hline
\end{tabular}




\begin{tabular}{|c|c|c|c|}
\hline \multicolumn{4}{|c|}{ Family: Aliaceae } \\
\hline 204. & Allium сера L. & $\mathrm{G}$ & Herb \\
\hline 205. & Allium sativum L. & $\mathrm{G}$ & Herb \\
\hline \multicolumn{4}{|c|}{ Family: Aspergaraceae } \\
\hline 206. & Asparagus adsendens Roxb. & $\mathrm{N}$ & Shrub \\
\hline \multicolumn{4}{|c|}{ Family: Cyperaceae } \\
\hline 207. & Cyperus nivens Retz. & $\mathrm{H}$ & Herb \\
\hline 208. & Cyperus nutans Vahl, Enum. & $\mathrm{H}$ & Herb \\
\hline \multicolumn{4}{|c|}{ Family: Dioscoreaceae } \\
\hline 209. & Dioscorea bulbifera Voigt. & $\bar{G}$ & Herb \\
\hline \multicolumn{4}{|c|}{ Family: Liliaceae } \\
\hline 210. & Tulipa stellata HK.f & $\mathrm{G}$ & Herb \\
\hline \multicolumn{4}{|c|}{ Family: Poaceae } \\
\hline 211. & Avena fatua L. & $\mathrm{CH}$ & Herb \\
\hline 212. & Bromus japonicus Thunb. ex Murr. & $\mathrm{TH}$ & Herb \\
\hline 213. & Cynodon dactytlon Pers. & $\mathrm{H}$ & Herb \\
\hline 214. & Dendrocalamus strictus (Roxb.)Nees. & $\mathrm{CH}$ & Herb \\
\hline 215. & Dendrcalamus parvifolium Dc. & $\mathrm{CH}$ & Herb \\
\hline 216. & Dendrocalamus polycarpum Dc. & $\mathrm{CH}$ & Herb \\
\hline 217. & Poa annua L. & $\mathrm{H}$ & Herb \\
\hline \multicolumn{4}{|c|}{ Gymnosperm } \\
\hline \multicolumn{4}{|c|}{ Family: Pinaceae } \\
\hline 218. & Pinus roxburghii Sarg. & $\mathrm{M}$ & Tree \\
\hline \multicolumn{4}{|c|}{ Pteridophytes } \\
\hline \multicolumn{4}{|c|}{ Family: Adiantaceae } \\
\hline 219. & Adiantum incisum Forssk. & $\mathrm{TH}$ & Pteridophyte \\
\hline \multicolumn{4}{|c|}{ Family: Dryopteradaceae } \\
\hline 220. & Dryopteris spp & $\mathrm{H}$ & Pteridophyte \\
\hline \multicolumn{4}{|c|}{ Family: Pteridaceae } \\
\hline 221. & Pteris cretica L. & $\mathrm{H}$ & Pteridophyte \\
\hline \multicolumn{4}{|c|}{ Family: Thelypteridiaceae } \\
\hline 222. & Cyclosorus prolifera Retz. & $\mathrm{TH}$ & Pteridophyte \\
\hline
\end{tabular}

TH=Therophytes; M=Macrophanerophytes; N=Nanophanerophytes; $\mathrm{CH}=$ Chamaephytes; H=Hemicryptophytes; HH=Hydrophytes; G=Geophytes; E=Epiphytes; L=Liana/Climber; AD=Angiosperm Dicot; AM=Angiosperm Monocot; PT=Pteridophyte; $\mathrm{G}=\mathrm{Gymnosperm}$. 
Among the families of angiosperms, Asteraceae has been found to be the dominant family with 20 species and 15 genera followed by Lamiaceae (14 species and 9 genera), Fabaceae (9 species and 9 genera), Rosaceae (9 species and 7 genera), Moraceae (9species and 2 genera), Euphorbiaceae (8 species and 4 genera), Poaceae ( 7 species and 5 genera), Solanaceae (7 species and 2 genera), Ranunculaceae (6 species and 4 genera), Amaranthaceae (5 species and 4 genera), Mimosaceae (5 species and 3 genera), Rhamnaceae (5 species and 3 genera), Convulvulaceae (5 species and 1 genera), Myrtaceae (4 species and 4 genera), Scrophulariaceae (4 species and 4 genera), Acanthaceae(4 species and 3 genera) and
Caryophyllaceae (4species and 2 genera). Ficus with 7 species has been recorded as the dominant genera of the study area and is followed by Euphorbia, Ipomoea and Solanum (5 species each), Acacia, Cassia, Dendrocalamus, Mentha, Trifolium, and Zizyphus (3 species each). Genera recorded with two species in the study area are Ajuga, Allium, Amaranthus, Bauhinia, Circium, Commelina, Cyperus, Datura, Geranium, Grewia, Indigofera, Justicia, Morus, Randia, Saussurea, Silene, Sonchus, Stellaria, Viola and Vitex.

The comparison of first five dominant families recorded in the study area with adjoining areas has been presented in Table-3.

Table- 2: Percentage and ratios of the families, genera and species of dicots and monocots, (excluding gymnosperms and pteridophytes)

\begin{tabular}{|c|c|c|c|c|c|c|c|}
\hline \multirow{2}{*}{ Taxa } & \multicolumn{2}{|c|}{ Dicots } & \multicolumn{2}{c|}{ Monocots } & \multirow{2}{*}{ Total } & \multicolumn{2}{c|}{ Ratio } \\
\cline { 2 - 7 } & $\begin{array}{c}\text { Total } \\
\text { Number }\end{array}$ & \%age & $\begin{array}{c}\text { Total } \\
\text { Number }\end{array}$ & \%age & & Dicots & Monocots \\
\hline Families & 69 & 86.25 & 6 & 0.75 & 80 & 11.5 & 1 \\
\hline Genera & 152 & 91.02 & 10 & 0.06 & 167 & 15.2 & 1 \\
\hline Species & 203 & 91.44 & 14 & 0.06 & 222 & 14.5 & 1 \\
\hline
\end{tabular}

Table-3: Comparison of dominant families of study area with adjoining areas.

\begin{tabular}{|c|l|l|l|l|l|l|l|}
\hline $\begin{array}{l}\text { S. } \\
\text { N } \\
\mathbf{0} .\end{array}$ & $\begin{array}{l}\text { Study area } \\
\text { (Author) }\end{array}$ & $\begin{array}{l}\text { Patnitop } \\
\text { Hills } \\
\mathbf{( K u m a r} \\
\text { K. 1997) }\end{array}$ & $\begin{array}{l}\text { Trikuta Hills } \\
\mathbf{( K o u r , ~ 2 0 0 1 )}\end{array}$ & $\begin{array}{l}\text { Kalakote } \\
\text { Range } \\
\text { (Singh 2002) }\end{array}$ & $\begin{array}{l}\text { Mahamaya } \\
\text { (Sudan, 2007) }\end{array}$ & $\begin{array}{l}\text { Mansar- } \\
\text { Surinsar } \\
\text { WLS (Rai, } \\
\mathbf{2 0 0 7 )}\end{array}$ & $\begin{array}{l}\text { Kishtwar } \\
\text { (Raina } \\
\text { and } \\
\text { Kumar, } \\
\text { 2011) }\end{array}$ \\
\hline 1. & Asteraceae & Asteraceae & Asteraceae & Asteraceae & Asteraceae & Fabaceae & Asteraceae \\
\hline 2. & Lamiaceae & Lamiaceae & Fabaceae & Lamiaceae & Poaceae & Poaceae & Fabaceae \\
\hline 3. & Fabaceae & Poaceae & Poaceae & Fabaceae & Fabaceae & Asteraceae & Lamiaceae \\
\hline 4. & Rosaceae & Fabaceae & Lamiaceae & Ranunculaceae & Lamiaceae & Lamiaceae & Rosaceae \\
\hline 5. & Moraceae & Rosaceae & Euphorbiaceae & Rosaceae & Amaranthaceae & Euphorbiaceae & Poaceae \\
\hline
\end{tabular}

Dominant families of the study area resembles with the adjoining region of Kalakote. Poaceae which find place in the dominant families in other areas could not find place in the first five dominant families of the present study area. Comparison of largest families and genera of plants of selected areas in the different natural regions give clue about the phyto-geographical variation within the state. The climatic conditions especially the availability of moisture, appears to be the main aspect bringing about the spatial phyto-geographical features (Singh, 2002) and this has also been observed in the 
present study area. Analysis of floristic composition areas has been presented in Table- 4. All these further revealed that the ratio of family to genera is $1: 2.87$, family to species is $1: 2.77$ and genera to species is $1: 1.32$. The comparative account of ratio of families to genera and genera to species of Lamberi forest range with some of the adjoining ratios have been observed to be lower as compared to the other regions and comparatively closer to Kalakote range which located adjoin to the study area. From the table it is also clear that these ratios are directly proportional to the biogeographic area.

Table 4: Comparison of Floristic Diversity of Study area with other areas

\begin{tabular}{|l|c|c|c|c|c|c|}
\hline Floristic diversity & F & G & S & $\begin{array}{c}\text { Ratio } \\
\text { (F:G) }\end{array}$ & $\begin{array}{c}\text { Ratio } \\
\text { (F:S) }\end{array}$ & $\begin{array}{c}\text { Ratio } \\
\text { (G:S) }\end{array}$ \\
\hline $\begin{array}{l}\text { Kalakote Range, J\&K } \\
\text { (Singh, 2002) }\end{array}$ & 99 & 253 & 356 & $1: 2.55$ & $1: 3.59$ & $1: 1.41$ \\
\hline $\begin{array}{l}\text { Mahamaya Forests, J\&K } \\
\text { (Sudan, 2007) }\end{array}$ & 73 & 192 & 282 & $1: 2.63$ & $1: 3.86$ & $1: 4.68$ \\
\hline $\begin{array}{l}\text { Kishtwar, J\&K } \\
\text { (Raina and Kumar, 2011) }\end{array}$ & 96 & 242 & 384 & $1: 2.52$ & $1: 4$ & $1: 1.59$ \\
\hline $\begin{array}{l}\text { Lamberi Forest Range, J\&K } \\
\text { (present work) }\end{array}$ & 80 & 167 & 222 & $1: 2.87$ & $1: 2.77$ & $1: 1.32$ \\
\hline
\end{tabular}

F= Family; G= Genera; $S=$ Species

\section{Conclusion}

The present study indicates that Lamberi forest range is very rich in phytodiversity. However, unplanned use, habitat destruction, grazing and some anthropogenic activities may lead to degradation of the area that may result in depletion of some of the species from area. The comprehensive information on species diversity of the Lamberi forest range may be of great help in developing a strategy and action plan for conservation management and sustainable utilization of its plant resources.

\section{References}

Bekele, T. 1994. Phytosociology and ecology of humid Afromontane forest in the Central Plateaus of Ethiopia. $\boldsymbol{J}$. veg. Sci, 5: 87-98.

Bulut, Z. and Yilmaz, H. 2010. The current situation of threathened endemic flora in Turkey: Kemaliye (Erzincan) CASE. Pak. J. Bot, 42: 711-719.

Farooquee, N.A. and Saxena, K.G. 1996. Conservation and utilization of medicinal plants in high hills of the central Himalayas. Environ. Conserv, 23: 75-80.

Giriraj, A., Murthy, M. and Ramesh, B. 2008. Vegetation composition, structure and patterns of diversity: a case study from the tropical wet evergreen forests of the Western Ghats, India. Edin. J. Bot, 65: 1-22.
Kilic, M. and Arslan, O.S. 2010. Turkey's Forests and Biodiversity. International Symposium on Biology of Rare and Endemic Plant Species. (Biorare Symposium) May 26-29.

Kour, I. 2001. Phyto-diversity and impact of tourism on the vegetation of Trikuta Hills(J\&K). Ph.D. Thesis submitted to University of Jammu, Jammu, J\&K, India.

Kumar, K. 1997. Studies on plant diversity of Patnitop and adjoining area and impact of biotic activities. Ph.D. Thesis submitted to University of Jammu, Jammu, J\&K, India.

Negi, K.S. and Gaur, R.D. 1994. Principal wild food plants of Western Himalaya, Uttar Pradesh, India. In: Higher plants of Indian subcontinent( Ed. Gupta B K). Bishen Singh Mahendra Pal Singh, Dehradun, India pp 1-78

Nune, S., Kassie, M. and Mungatana, E. 2010. Forestry resource accounting: the experience of Ethiopia. CEEPA Discussion Paper No 47, Centre for Environmental Economics and Policy in Africa, University of Pretoria, South Africa

Pappoe, A. N., Armah, F.A., Quaye, E.C., Kwakye, P.K. and Buxton, G.N.2010. Composition and stand structure of a tropical moist semideciduous forest in Ghana. Int. Res. J. Plant Sci, 1: 095-106.

Rai, A. 2007. Studies on Phanerogan Diversity of MansarSurinsar Wildlife Sanctuary, J\&K. Ph.D. Thesis submitted to University of Jammu. Jammu, J\&K. India. 
Raina, A.K. and Kumar, R. 2011. Floristic composition, lifeform classification and biological spectrum of the catchment of Rattle H.E. project, District Kishtwar- J\&K. Environment conservation Journal, 12(3): 1-6

SCBD: Secretariat of the Convention on Biological Diversity 2001. The Value of Forest Ecosystems. Montreal, SCBD, 67p. (CBD Technical Series no. 4).

Sharma, N. 2003. Biodiversity characterization at landscape level using RS and GIS in District, Jammu. Ph.D. Thesis submitted to University of Jammu, Jammu, J\&K, India.

Singh, J. 2002. Phyto-diversity of Kalakote Range (Rajouri, $\mathrm{J} \& \mathrm{~K}$ ) and impact of mining and nomadism on the vegetation. Ph.D. Thesis submitted to University of Jammu, Jammu, J\&K, India.
Sudan, J. 2007. Phytodiversity and Socio-economic studies of Mahamaya Catchment, Jammu, J\&K. Ph.D. Thesis submitted to University of Jammu, Jammu, J\&K, India.

Venu, P. 2002. Some conceptual and practical issues in taxonomic research. Curr. Sci., 82(8): 924-933

Villasenor, J. L., Maeda, P., Rosell, J.A. and Ortiz, E. 2007 Plant families as predictors of plant biodiversity in Mexico. Divers. Dist., 13: 871-876.

Vivero, J.L., Kelbessa, E. and Demissew, S. 2005. The Red List of Endemic Trees and Shrubs of Ethiopia and Eritrea. Fauna and Flora International. Cambridge, U.K. 